\title{
Aplicación de la dinámica de sistemas en la identificación y evaluación de las potencialidades económicas para mejorar el desarrollo de la provincia de Castrovirreyna, Huancavelica Application of dynamical systems in the identification and evaluation of economic potentialities to improve development in the province of Castrovirreyna, Huancavelica
}

\author{
Javier F Márquez Camarena' \\ imccamarena@hotmail.com \\ Víctor R. Rodríguez P.eña ${ }^{2}$ \\ Universidad Nacional de Huancavelica \\ covi_rodriguez@hotmail.com

\section{Raúl P. Meza Cárdenas ${ }^{3}$} \\ Universidad Nacional de Huancavelica \\ primifeunh@hotmail.com
}

\section{RESUMEN}

Esta investigación ha permitido aplicar la dinámica de sistemas para identificar y evaluar las potencialidades económicas de la provincia de Castrovirreyna en el departamento de Huancavelica con el fin de mejorar su desarrollo, asimismo determinar los factores y la información sistematizada que influyen en la identificación y evaluación. Este trabajo fue de alcance descriptivo no experimental. Fueron realizadas 250 encuestas y entrevistas a la población y autoridades con la finalidad de obtener información de las riquezas naturales que posee la provincia y se procedió a la simulación para determinar la evaluación de las potencialidades de la provincia de Castrovirreyna. Algunos resultados son que, el análisis inicial muestra el descenso del potencial económico, debido a factores como carencia de cultura de los pobladores, poca atención de las autoridades, falta de desarrollo de información (95\%), desconocimiento en la producción de las riquezas $(70 \%)$, pocas vías de acceso (40\%), pocos canales de irrigación (30 $\%)$. Con la mejora de los factores en mención en el modelo se observa el incremento de las riquezas como agricultura $25 \%$, minería $23 \%$, ganadería $19 \%$, pesquería $26 \%$ y turismo $14 \%$, en el cuarto año, lo que permitiría mejorar el desarrollo de la provincia. En conclusión, con la integración de las riquezas se incrementa sustancialmente el potencial económico de $20 \%$ a $826 \%$ durante el cuarto año, el cual repercute positivamente en la mejora de la calidad de vida de los pobladores.

Palabras clave: Modelo, agricultura, actividad económica, análisis de sistemas, desarrollo, factor, producción, sistemas, información.

\section{ABSTRACT}

This research paper has allowed to apply the dynamical systems to identify and evaluate the economic potentialities in the province of Castrovirreyna, department of Huancavelica in order to improve its development. At the same time, it helps to determine what factors and systematic information influence in the identification and assessment of it. This study was non experimental of descriptive scope. To obtain information from the natural resources of the province, 250 surveys and interviews with people and authorities were conducted followed by a simulation to assess the Castrovirreyna potential. This initial analysis shows the decline of the economic potential due to factors such as people's poor instruction, indifferent authorities, lack of information ( $95 \%)$, poor knowledge of their own natural resources (70\%), few access roads (40\%), and few irrigation canals $(30 \%)$. In the fourth year the model showed an improvement of the mentioned factors by an increase of $25 \%$ in agriculture, $23 \%$ in mining, $19 \%$ in livestock, $26 \%$ in fishery and $14 \%$ in tourism, which would enhance the development of the province. Finally, with an integrated management of productive activities, the economic potential of Castrovirreyna province would substantially increase from $20 \%$ to $826 \%$, showing a positive effect on improving the life quality of the population.

Keywords: Model, agriculture, economic activity, systems analysis, development, factor, production, systems, information.

Historial del artículo:

Recibido: 14 de diciembre de 2014. Aprobado: 23 de marzo 2015. Disponible en línea: 30 de junio de 2015

1 Mg. en Administración, mención Informática para la Gestión. Ing. de Sistemas, docente en la Universidad Nacional de Huancavelica.

2 Ing. de Sistemas, docente en la Universidad Nacional de Huancavelica.

3 Docente de la Universidad Nacional de Huancavelica. 


\section{INTRODUCCIÓN}

Muchas instituciones se ven disminuidas en su rendimiento por falta de un buen sistema de información. La provincia de Castrovirreyna, Huancavelica, no es ajena a ello, por tal razón tiene alto índice de desnutrición en la población. Castellanos define que un sistema de información (SI) es un conjunto interrelacionado de elementos que proveen información para el apoyo de las funciones de operación, gerencia y toma de decisiones en una organización (1). Laudon \& Laudon establecen que la organización debe estar consciente y abierta a las influencias de los sistemas de información, para beneficiarse de las nuevas tecnologías (2).

Por los viajes realizados a Castrovirreyna y la información obtenida, se constató la gran existencia de recursos naturales en dicha provincia, los cuales no son la extrema pobreza de la población de la provincia de Castrovirreyna, situación inadmisible porque cuenta con riquezas naturales, según información obtenida del estudio con proyección al futuro del distrito de Arma de la provincia (4).

El problema planteado fue: ¿̇Cómo la aplicación de la dinámica de sistemas, con el diseño de un modelo, determina la identificación y evaluación de los recursos naturales de la provincia de Castrovirreyna, Huancavelica? Aracil \& Gordillo exponen los elementos básicos del lenguaje sistemático utilizando ejemplos concretos (5).

El objetivo fue diseñar modelos mediante la aplicación de la dinámica de sistemas para identificar y evaluar las riquezas naturales que posee la provincia. García sostiene que la dinámica de sistemas es la metodología que permite introducir nuestra percepción

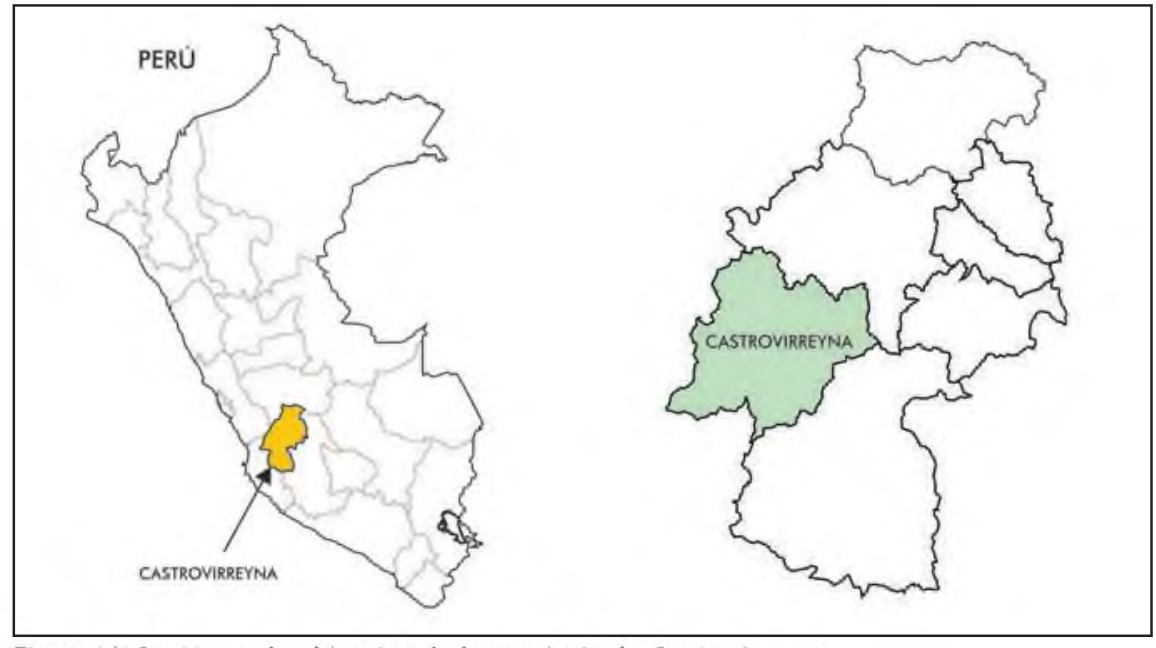

Figura $N^{\circ}$ 1: Mapa de ubicación de la provincia de Castrovirreyna.

utilizados debido al conformismo y desconocimiento de la población, así como por el poco apoyo de las autoridades.

En el desarrollo de este trabajo se logró la información necesaria in situ con entrevistas realizadas a la población para identificar y evaluar las potencialidades económicas de la provincia. Kendall \& Kendall mencionan que una entrevista necesitará crear, en forma rápida, un clima de confianza y entendimiento, pero sin perder el control de la entrevista. También necesita promover su sistema, planteando a su entrevistado toda la información necesaria (3).

El $80 \%$ de la población tiene recelo de los estudios que se realizan en la provincia porque mencionan que no se cumplen. Con la información obtenida se identificó y evaluó las potencialidades económicas de la provincia. El trabajo de investigación se desarrolló considerando de la realidad en un ordenador (6). La realidad de la provincia de Castrovirreyna los pobladores carecen de vías de comunicación necesarias, así como adolecen de poca educación y cultura, tienen poca importancia de las riquezas naturales que les rodean, condiciones que llevan a realizar este estudio.

La hipótesis planteada fue que la aplicación de la dinámica de sistemas en el diseño de un modelo determina la identificación y evaluación de las potencialidades económicas para mejorar el desarrollo de la provincia de Castrovirreyna, Huancavelica.

En esta hipótesis se muestra el principio del diagrama de Forrester donde se utilizó gráficamente las variables como niveles, flujos y tasas, se validó el modelo, se observó la evolución temporal de las variables y se realizó el análisis respectivo de cada riqueza natural de la provincia. Ogata menciona que la obtención de 


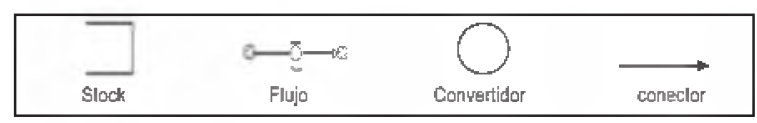

Figura $\mathrm{N}^{\circ}$ 2: Bloques de construcción usados en los modelos.

un modelo razonable del sistema completo constituye la parte básica de todo el análisis. Una vez que se dispone de tal modelo pueden usarse para el análisis diferentes técnicas analíticas y por computadora (6). Para dar inicio a la evaluación, fue considerada la producción anual de cada riqueza: la agricultura tuvo una producción de $521412 \mathrm{t}$; la minería, $7338 \mathrm{t}$; la pesquería, 751 t; ganadería, 168787 cabezas. El turismo registra en promedio un flujo anual de 515 personas.

\section{MATERIAL Y MÉTODOS}

El nivel de investigación fue descriptivo no experimental. La zona de estudio comprende la provincia de Castrovirreyna, región Huancavelica (figura $\mathrm{N}^{\circ} 1$ ), posee una extensión territorial de $3984,62 \mathrm{~km} 2$. Fueron realizadas 250 encuestas y entrevistas a la población y autoridades con la finalidad de obtener información de las riquezas naturales que posee la provincia; también se realizó visitas a los lugares turísticos como los molinos coloniales conocidos como "trapiche", ubicados al norte y a 10 minutos de la ciudad de Castrovirreyna.

En el presente trabajo se utilizó el software Stella con los diagramas de Forrester, representando con bloques de construcción al stock, flujo, convertidor y conector (figura $N^{\circ}$ 2). Según Méndez, pretende acercarse a la realidad de las relaciones causales por los cuales opera el sistema (7).

\section{RESULTADOS}

La tabla $N^{\circ} 1$ muestra los porcentajes de incremento anual en la producción de las potencialidades económicas de la provincia de Castrovirreyna, Huancavelica. Como referencia se tiene al inicio de la evaluación del sector agrario la cantidad de 52141 $\dagger$ de producción, que se incrementa en un promedio de $27 \%$ al final del cuarto año. El caso es explicado por la reducción de los obstáculos en el incremento de vías de comunicación y de construcciones de canales de irrigación ya que la provincia cuenta con ingentes recursos hídricos.

El análisis inicial muestra el descenso del potencial económico, debido a factores como carencia de cultura de los pobladores, poca atención de las autoridades, falta de desarrollo de información 195 $\%)$, desconocimiento en la producción de las riquezas (70\%), pocas vías de acceso $(40 \%)$, pocos canales de irrigación (30\%).

Con tales factores fueron construidos los modelos considerando la producción agraria anual de 52141 t (8), recursos hídricos de 245 millones de m3 (9) y 65000 ha de terreno (8), (figuras 3 y 4). Cervantes $\&$ Chiapa realizaron modelos más comunes que se aplican en los sistemas como modelo exponencial, logístico, estímulo-respuesta, autorreferencia y modelo buscando objetivo (10).

Tabla $N^{\circ}$ 1: Porcentaje de incremento del potencial económico y las riquezas de la provincia de Castrovirreyna, Huancavelica.

\begin{tabular}{ccccccc}
\hline $\begin{array}{c}\text { Período } \\
\text { tiempo } \\
\text { años }\end{array}$ & $\begin{array}{c}\text { Potencial } \\
\text { económico }\end{array}$ & $\begin{array}{c}\text { Producción } \\
\text { agraria }\end{array}$ & $\begin{array}{c}\text { Producción } \\
\text { minera }\end{array}$ & $\begin{array}{c}\text { Producción } \\
\text { ganadera }\end{array}$ & $\begin{array}{c}\text { Producción } \\
\text { pesquera }\end{array}$ & Turismo \\
\hline 0,00 & 0,20 & 0,25 & 0,30 & 0,40 & 0,40 & 0,10 \\
0,25 & 0,56 & 0,28 & 0,32 & 0,43 & 0,43 & 0,12 \\
0,50 & 0,94 & 0,31 & 0,34 & 0,45 & 0,47 & 0,13 \\
0,75 & 1,36 & 0,34 & 0,37 & 0,47 & 0,49 & 0,14 \\
1,00 & 1,79 & 0,36 & 0,39 & 0,49 & 0,52 & 0,15 \\
1,25 & 2,25 & 0,38 & 0,42 & 0,51 & 0,54 & 0,16 \\
1,50 & 2,74 & 0,40 & 0,44 & 0,52 & 0,56 & 0,17 \\
1,75 & 3,24 & 0,42 & 0,46 & 0,53 & 0,57 & 0,18 \\
2,00 & 3,75 & 0,43 & 0,48 & 0,54 & 0,59 & 0,19 \\
2,25 & 4,28 & 0,44 & 0,49 & 0,55 & 0,60 & 0,20 \\
2,50 & 4,82 & 0,46 & 0,50 & 0,56 & 0,62 & 0,21 \\
2,75 & 5,38 & 0,47 & 0,51 & 0,57 & 0,63 & 0,21 \\
3,00 & 5,94 & 0,48 & 0,51 & 0,57 & 0,64 & 0,22 \\
3,25 & 6,51 & 0,48 & 0,52 & 0,58 & 0,64 & 0,23 \\
3,50 & 7,08 & 0,49 & 0,52 & 0,58 & 0,65 & 0,23 \\
3,75 & 7,67 & 0,50 & 0,53 & 0,59 & 0,66 & 0,24 \\
4,00 & 8,26 & 0,50 & 0,53 & 0,59 & 0,66 & 0,24 \\
\hline
\end{tabular}




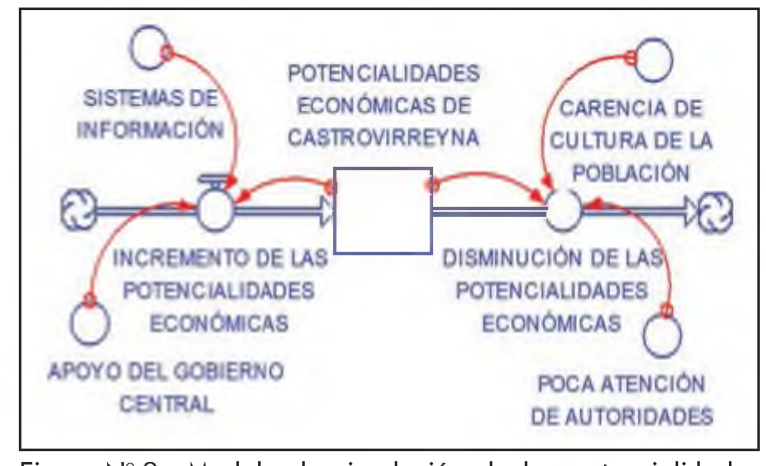

Figura $N^{\circ} 3$ : Modelo de simulación de las potencialidades económicas de la provincia de Castrovirreyna.

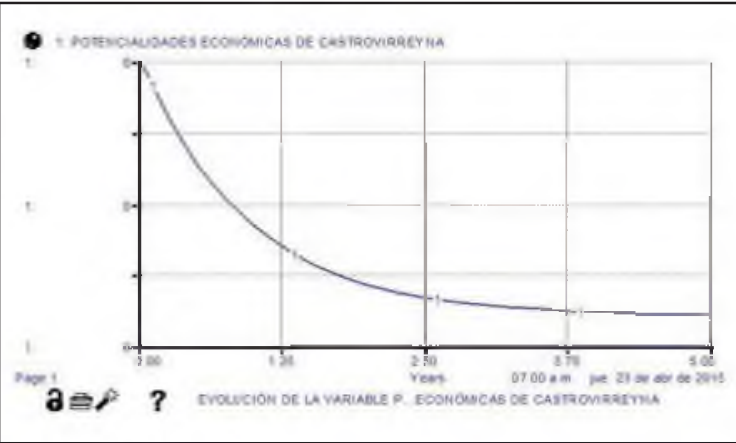

Figura $\mathrm{N}^{\circ}$ 4: Comportamiento de la variable potencialidades económicas de la provincia.

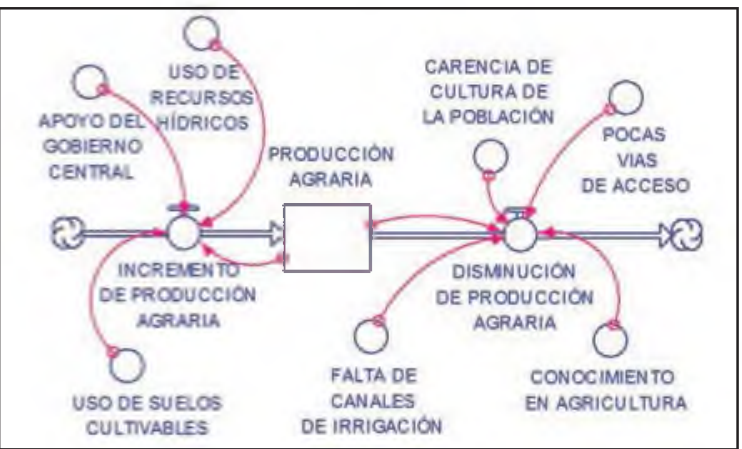

Figura $\mathrm{N}^{\circ}$ 5: Modelo de simulación del sector agricultura.

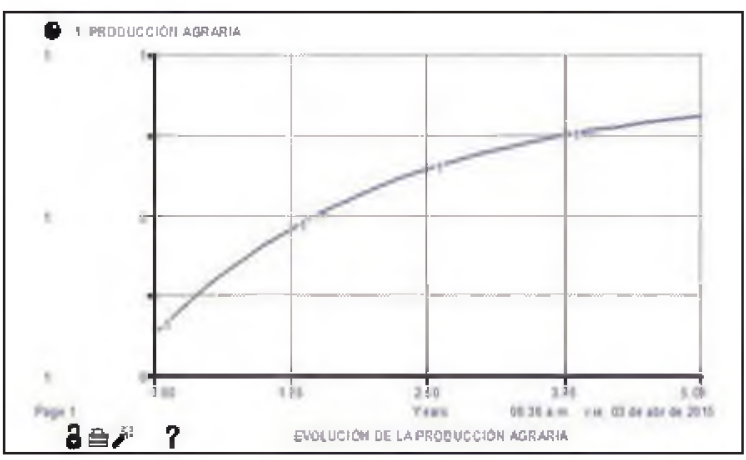

Figura $N^{\circ}$ 6: Evolución de la producción agraria mejorando las variables de disminución de la producción.

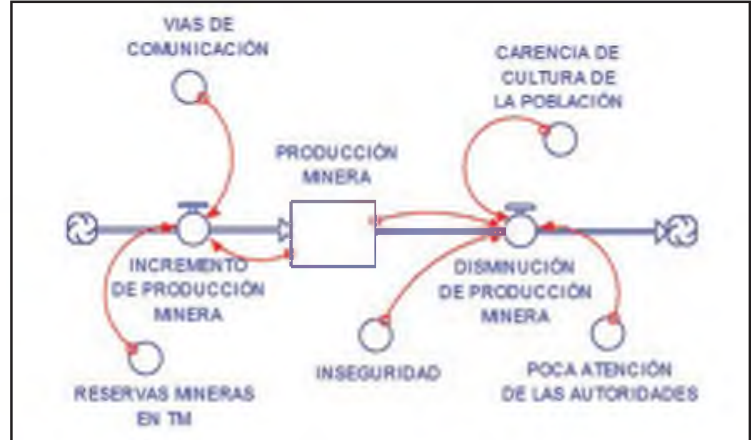

Figura $N^{\circ} 7$ : Modelo de simulación de la producción minera.

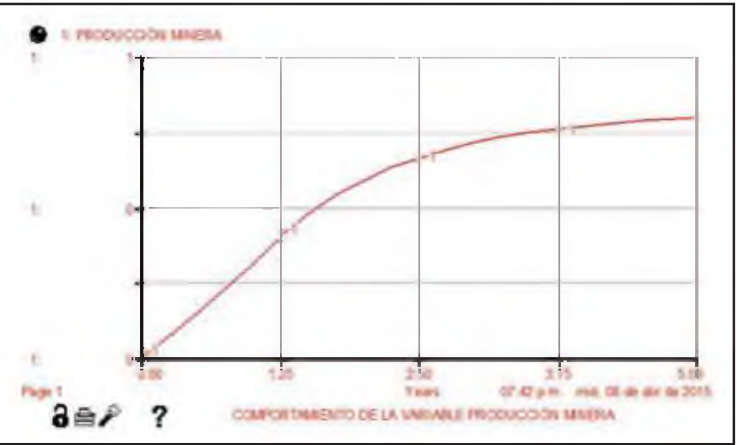

Figura $\mathrm{N}^{\circ} 8$ : Evolución de la producción minera.

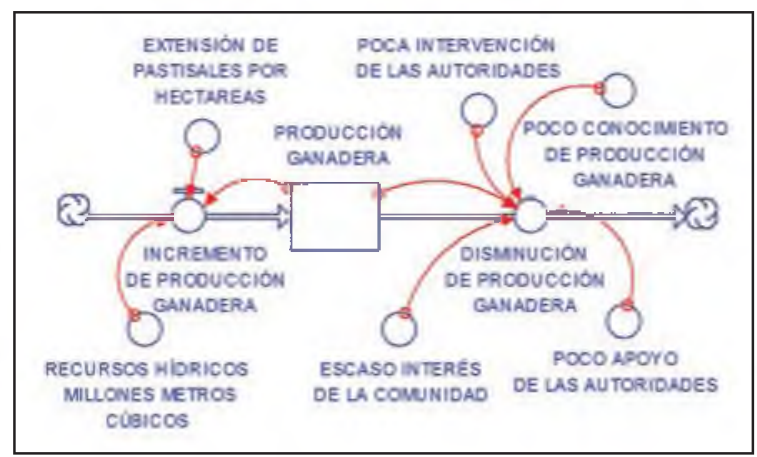

Figura №9: Modelo de simulación de la producción ganadera.

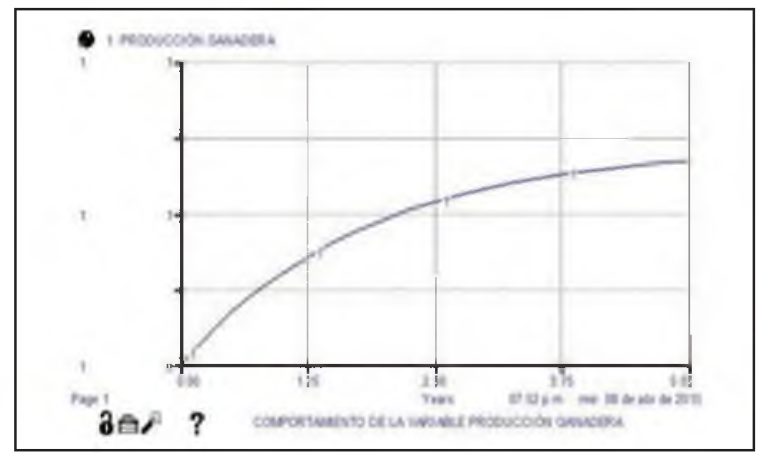

Figura $N^{\circ} 10$ : Evolución de la producción ganadera. 


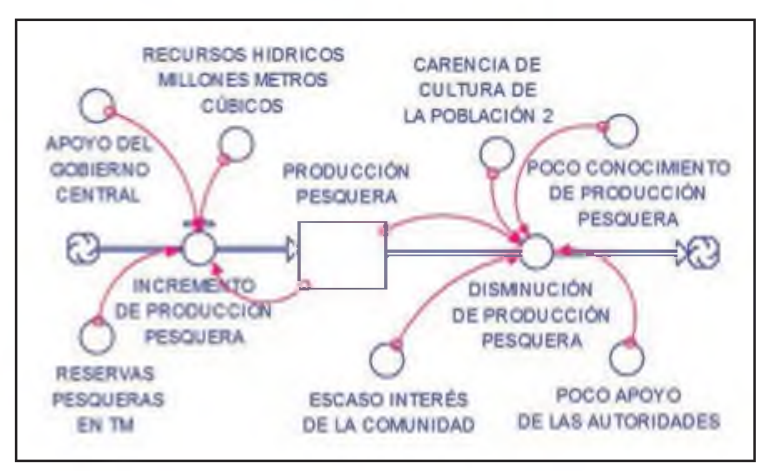

Figura $\mathrm{N}^{\circ}$ 11: Modelo de simulación de la producción pesquera.

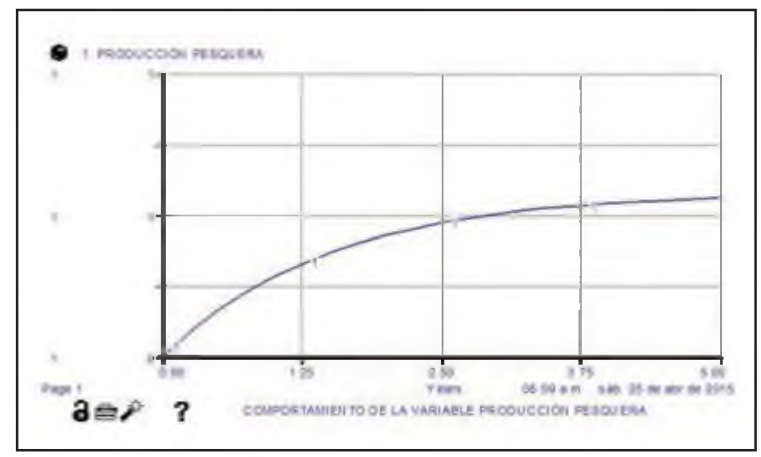

Figura $N^{\circ} 12$ : Evolución de la producción pesquera.

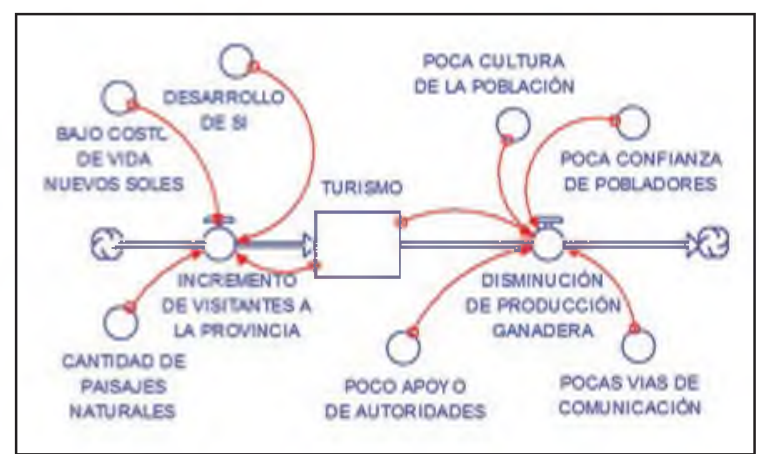

Figura $N^{\circ}$ 13: Modelo de simulación del turismo.

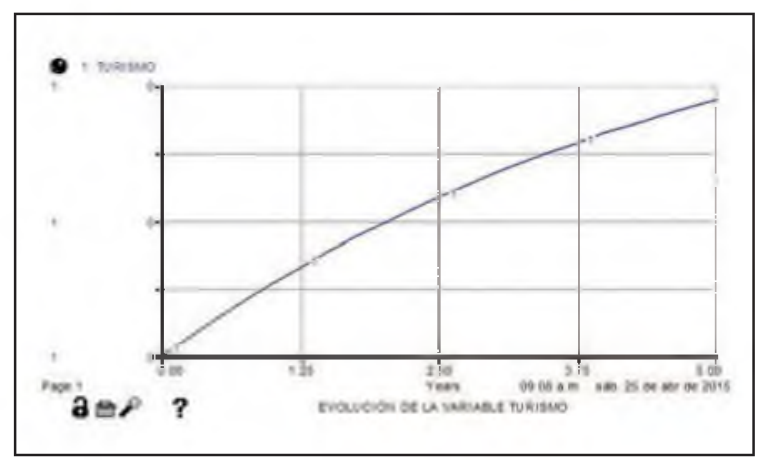

Figura $N^{\circ}$ 14: Evolución del incremento de turismo.
Con la debida atención en la producción agraria con el apoyo del gobierno central, uso adecuado de los recursos hídricos y la construcción de canales de irrigación se incrementa la producción (figuras 5 y 6). Para la Minería se consideró como referencia la producción de la empresa minera Castrovirreyna, ubicada en el distrito de Santa Ana de la provincia, con una producción anual de $7338+$ de concentrado cuyas reservas son $3049307+(11)$. Con la construcción de vías de acceso, atención de las autoridades y mayor seguridad se incrementa la producción minera (figuras 7 y 8$)$.

Para el rubro de la ganadería se consideró la producción anual de 168787 cabezas de ganado entre vacuno, caprino, porcino, equino y camélidos (11), extensión de pastizales de 142365 ha y el uso de los recursos hídricos (70\%), las cuales incrementan la producción ganadera (figuras 9 y 10 ).

Para el sector pesquería se consideró la producción de 751 † anuales de truchas y 245 millones de m3 (9) de recursos hídricos, que mediante la intervención del gobierno central aumenta la producción de truchas en la provincia (figuras 11 y 12).

Para el rubro del turismo se estimó la visita de un aproximado de 515 turistas (información obtenida de la Municipalidad Provincial de Castrovirreyna). La provincia tiene un bajo costo de vida, cuenta con 45 paisajes naturales que, desarrollando sistemas de información, se tiene un incremento bastante pronunciado de concurrencia de turistas en la provincia (figuras 13 y 14).

Con los incrementos de los recursos, considerando las variables: apoyo de gobierno central, construcción de vías de acceso, conocimiento de la producción y el desarrollo de sistemas, se mejora las potencialidades económicas de la provincia (figura 15).

\section{DISCUSIÓN}

La provincia de Castrovirreyna posee ingentes recursos hídricos de las lagunas, las cuales no son aprovechadas en la pesquería y la agricultura. En este caso las autoridades deben intervenir con apoyo decidido para mejorar la producción en los rubros mencionados.

La población argumenta que la provincia no cuenta con las vías de acceso necesarias para poder extraer sus productos del campo hacia la ciudad. Esta situación conlleva a reflexionar a todas las autoridades sobre la necesidad de otorgarles apoyo decidido.

Con la integración de los recursos naturales se observa el fuerte incremento sostenido del potencial económico, ya que en ello se considera el apoyo de las 


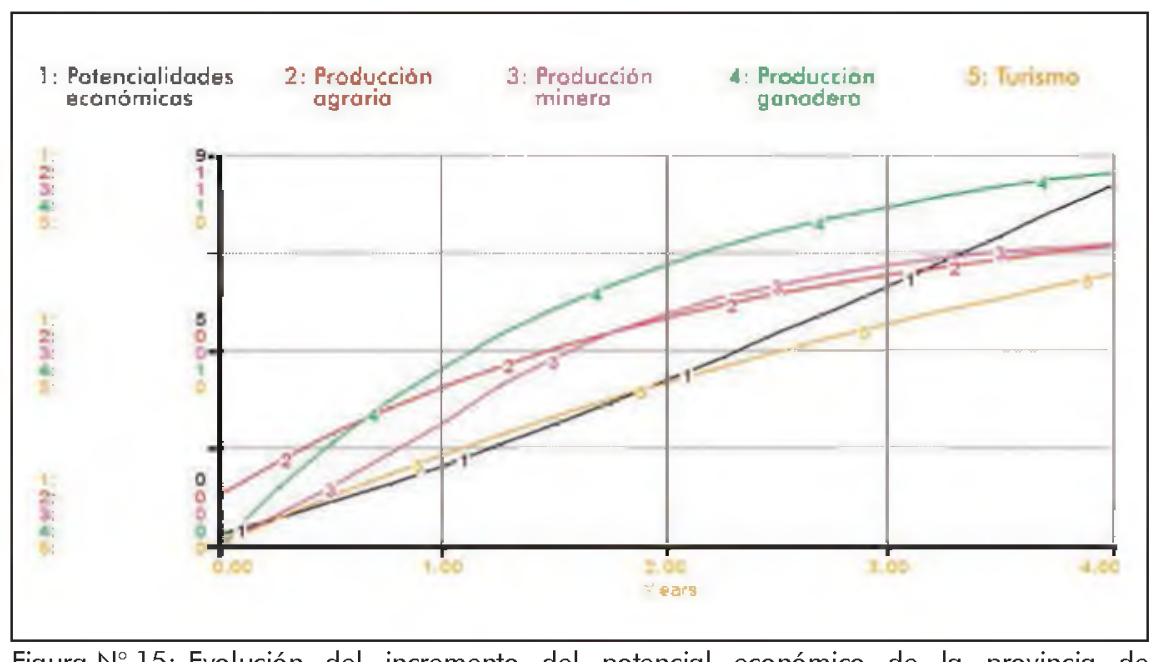

Figura $\mathrm{N}^{\circ}$ 15: Evolución del incremento del potencial económico de la provincia de Castrovirreyna, Huancavelica.

autoridades, la mejora de la cultura de la población, construcción de vías de acceso, así como el desarrollo de sistemas de información.

Entre las conclusiones podemos destacar que, se diseñó un modelo para evaluar y mejorar las potencialidades económicas de la provincia, demostrando que con el incremento de los recursos agrarios, mineros, ganaderos, pesqueros y turísticos, el potencial económico se incrementa de $20 \%$ a $826 \%$ al final del cuarto año.

Los factores como la construcción de más vías de comunicación (incremento del $70 \%$ ), el apoyo de gobierno central (90\%), la mejora de la cultura de la población $(70 \%)$, la construcción de canales de irrigación (70 \%) y la mayor seguridad (75\%) influyen positivamente en la evaluación y mejora de las potencialidades económicas de la provincia.

El desarrollo de sistemas de información es fundamental para el incremento del turismo, como también para la toma de decisiones en la producción de recursos; así, cuando se incrementa los sistemas de información de $5 \%$ a $100 \%$ se tiene un aumento en el turismo de 10 $\%$ a $24 \%$, y el potencial económico mejora de $20 \%$ hasta $826 \%$.

Los factores como la limitada educación (70\%), cultura $(80 \%)$ y escaso conocimiento de los pobladores sobre producción (75\%), repercuten negativamente en la producción de las riquezas de la provincia.

\section{Agradecimientos}

A la Universidad Nacional de Huancavelica que mediante el FOCAM hizo realidad esta investigación; a los doctores Alberto Un Han y José Franco Yépez, quienes apoyaron en forma incondicional en el estudio.

\section{REFERENCIAS BIBLIOGRÁFICAS}

1. Castellanos L. Desarrollo de sistemas de información, bajo un enfoque incremental. Maracaibo: Universidad Nacional Experimental de la Fuerza Armada; $2011 ; 75$ p.

2. Laudon K \& Laudon J. Sistemas de información gerencial. 12@ ed. México: Pearson Educación; 2013.

3. Kendall K \& Kendall J. Análisis y diseño de sistemas. 8a ed. México: Prentice Hall; 2012.

4. Desco. Plan estratégico concertado para el desarrollo del distrito de Arma, provincia de Castrovirreyna 2004-2020. Lima: Desco; 2009.

5. Aracil J \& Gordillo F. Dinámica de sistemas. España: Alianza Universidad Textos; 1997; II:21.

6. Ogata K. Dinámica de sistemas. México: Prentice Hall; 1987; I: 5

7. Méndez G. Dinámica de sistemas. Colombia: Editorial Universidad Distrital Francisco José de Caldas; 2012.

8. Gobierno Regional de Huancavelica. Compendio estadístico agrario de la región Huancavelica 2000 - 2009. Huancavelica: Dirección Regional Agraria, Gobierno Regional de Huancavelica; 2011 ; tomol.

9. Gobierno Regional de Huancavelica. Implementación del Plan de Desarrollo Acuícola año 2010. Huancavelica: Dirección Regional de la Producción, Gobierno Regional de Huancavelica; $2011 ; 16$ p.

10. Cervantes A \& Chiapa X. Numo Simoes. Modelación dinámica, modelos más comunes con Stella. Minakotsu. Lima; 2007; II:12-26.

11. Castrovirreyna Compañía Minera. Memoria anual 2010. Lima; $2011 ; 53 \mathrm{p}$. 